\title{
Supporting Information for A Coarse-Grained Model for Microbial Lipopeptide Surfactin and Its Application in Self-Assembly
}

Hongze Gang, ${ }^{1,3}$ Hao He, ${ }^{1}$ Zhou Yu, ${ }^{1}$ Zhenyu Wang, ${ }^{1}$ Jinfeng Liu, ${ }^{1,3}$ Xiujuan He, ${ }^{2}$

Xinning Bao, ${ }^{1,2}$ Yingcheng $\mathrm{Li},{ }^{*, 2}$ and Bozhong $\mathrm{Mu}^{*}, 1,3$

1 State Key Laboratory of Bioreactor Engineering and School of Chemistry and Molecular Engineering, East China University of Science and Technology, Meilong 130, 200237 Shanghai, P.R. China

${ }^{2}$ Sinopec Key Lab of Surfactants for EOR, Sinopec Shanghai Research Institute of Petrochemical Technology, North Pudong 1658, 201208 Shanghai, P.R. China

${ }^{3}$ Engineering Research Center of Microbial Enhanced Oil Recovery, MOE, East China University of Science and Technology, Meilong 130, 200237 Shanghai, P.R. China 
Table S1. Detail Parameters of all the Atomistic and CG Simulations.

\begin{tabular}{|c|c|c|c|}
\hline System & surfactin & $\mathrm{Na}^{+}$ & Water \\
\hline AAI & 50 & 100 & 6127 (SPC) \\
\hline $\mathrm{CGI}_{\mathrm{I}}$ & 50 & 100 & $1570(\mathrm{~W})$ \\
\hline $\mathrm{CGI}_{\mathrm{II}}$ & 50 & 100 & $1570(\mathrm{~W})$ \\
\hline $\mathrm{CGI}_{\text {III }}$ & 50 & 100 & $1570(\mathrm{~W})$ \\
\hline $\mathrm{CGI}_{\mathrm{IV}}$ & 50 & 100 & $1570(\mathrm{~W})$ \\
\hline AAM & 25 & 50 & 8512 (SPC) \\
\hline $\mathrm{CGM}_{\mathrm{I}}$ & 25 & 50 & $2894(\mathrm{~W})$ \\
\hline $\mathrm{CGM}_{\mathrm{II}}$ & 25 & 50 & $2915(\mathrm{~W})$ \\
\hline $\mathrm{CGM}_{\text {III }}$ & 25 & 50 & $2902(\mathrm{~W})$ \\
\hline $\mathrm{CGM}_{\mathrm{IV}}$ & 25 & 50 & $2912(\mathrm{~W})$ \\
\hline BA & 25 & 50 & $13527(\mathrm{~W})$ \\
\hline $\mathrm{BA}_{\mathrm{I}}$ & 200 & 400 & $129924(\mathrm{~W})$ \\
\hline $\mathrm{BA}_{\mathrm{II}}$ & 200 & 400 & $129903(\mathrm{~W})$ \\
\hline $\mathrm{BA}_{\mathrm{III}}$ & 200 & 400 & $129882(\mathrm{~W})$ \\
\hline $\mathrm{BA}_{\mathrm{IV}}$ & 300 & 600 & $128799(\mathrm{~W})$ \\
\hline $\mathrm{BA}_{V}$ & 300 & 600 & $128790(\mathrm{~W})$ \\
\hline $\mathrm{BA}_{\mathrm{VI}}$ & 300 & 600 & $128775(\mathrm{~W})$ \\
\hline BAvII & 400 & 800 & $127672(\mathrm{~W})$ \\
\hline BAvIII & 400 & 800 & $127651(\mathrm{~W})$ \\
\hline BA IX $_{\text {IX }}$ & 400 & 800 & $127670(\mathrm{~W})$ \\
\hline
\end{tabular}


Table S2. Gaussian Fitting of the Density Distributions of Surfactin Monolayers at the Octane/Water Interface.

\begin{tabular}{ccc}
\hline System & $\rho_{\max }\left(\times 10^{-3} \mathrm{~g} / \mathrm{cm}^{3}\right)$ & $\sigma(\mathrm{nm})$ \\
\hline AAI & $968.7 \pm 19.4$ & $1.34 \pm 0.03$ \\
CGI $_{I}$ & $1175.3 \pm 3.4$ & $1.07 \pm 0.00$ \\
CGI $_{\text {II }}$ & $1189.9 \pm 8.7$ & $1.07 \pm 0.01$ \\
CGI $_{\text {III }}$ & $1111.5 \pm 0.4$ & $1.14 \pm 0.00$ \\
CGI $_{\text {IV }}$ & $1122.3 \pm 5.4$ & $1.14 \pm 0.01$ \\
\hline
\end{tabular}


Table S3. Bond Interaction Parameters for CG Simulations of Pre-Assembled

Surfactin Monolayer at the Octane/Water Interface.

\begin{tabular}{|c|c|c|c|c|c|c|c|c|c|c|c|}
\hline \multirow[b]{2}{*}{$i-j$} & \multirow[b]{2}{*}{ CGI I } & \multicolumn{2}{|c|}{$d_{\mathrm{ij}}(\mathrm{nm})$} & & \multirow[t]{2}{*}{$K_{\mathrm{d}}(\mathrm{kJ} / \mathrm{mol})$} & \multirow[b]{2}{*}{$i-j-k$} & \multicolumn{4}{|c|}{$\theta$ (degree) } & \multirow[t]{2}{*}{$K_{\theta}(\mathrm{kJ} / \mathrm{mol})$} \\
\hline & & $\mathrm{CGI}_{\text {II }}$ & $\mathrm{CGI}_{\text {III }}$ & CGIIv & & & $\mathrm{CGI}_{\mathrm{I}}$ & $\mathrm{CGI}_{\text {II }}$ & $\mathrm{CGI}_{\mathrm{III}}$ & CGIIv & \\
\hline $1-2$ & 0.4 & 0.291 & 0.4 & 0.291 & 5000 & $2-1-4$ & 100 & 100 & 120.58 & 121 & 25 \\
\hline $1-4$ & 0.35 & 0.34 & 0.35 & 0.34 & 200 & $2-1-17$ & 100 & 100 & 96.5 & 108 & 25 \\
\hline $1-17$ & 0.318 & 0.31 & 0.318 & 0.31 & 1250 & $4-1-17$ & 127 & 127 & 111.81 & 104 & 25 \\
\hline $4-5$ & 0.33 & 0.33 & 0.33 & 0.33 & 7500 & $1-4-5$ & 100 & 100 & 96.1 & 105 & 25 \\
\hline $4-6$ & 0.35 & 0.307 & 0.35 & 0.307 & 200 & $1-4-6$ & 127 & 127 & 84.24 & 95 & 25 \\
\hline $6-7$ & 0.33 & 0.334 & 0.33 & 0.334 & 7500 & $5-4-6$ & 100 & 100 & 156.18 & 153 & 25 \\
\hline $6-8$ & 0.35 & 0.367 & 0.35 & 0.367 & 200 & $4-6-7$ & 100 & 100 & 101.17 & 104 & 25 \\
\hline $8-10$ & 0.35 & 0.313 & 0.35 & 0.313 & 200 & $4-6-8$ & 127 & 127 & 115.7 & 109 & 25 \\
\hline $10-11$ & 0.32 & 0.251 & 0.32 & 0.251 & 7500 & $7-6-8$ & 100 & 100 & 116.28 & 113 & 25 \\
\hline $10-13$ & 0.35 & 0.329 & 0.35 & 0.329 & 200 & $6-8-9$ & 100 & 100 & 97.9 & 96 & 25 \\
\hline $13-14$ & 0.33 & 0.337 & 0.33 & 0.337 & 7500 & $6-8-10$ & 127 & 127 & 101.5 & 88 & 25 \\
\hline $13-15$ & 0.35 & 0.37 & 0.35 & 0.37 & 200 & $9-8-10$ & 100 & 100 & 166.73 & 162 & 25 \\
\hline $15-16$ & 0.33 & 0.329 & 0.33 & 0.329 & 7500 & 8-10-11 & 100 & 100 & 93.05 & 112 & 25 \\
\hline $15-18$ & 0.253 & 0.238 & 0.253 & 0.238 & 1250 & 8-10-13 & 127 & 127 & 107.67 & 117 & 25 \\
\hline $17-18$ & 0.27 & 0.261 & 0.27 & 0.261 & 1250 & $11-10-13$ & 100 & 100 & 116.26 & 115 & 25 \\
\hline $18-19$ & 0.33 & 0.347 & 0.33 & 0.347 & 7500 & $10-13-14$ & 100 & 100 & 105.88 & 101 & 25 \\
\hline $19-20$ & 0.47 & 0.477 & 0.47 & 0.477 & 1250 & $10-13-15$ & 127 & 127 & 109.13 & 115 & 25 \\
\hline $20-21$ & 0.47 & 0.449 & 0.47 & 0.449 & 1250 & $14-13-15$ & 100 & 100 & 120.02 & 122 & 25 \\
\hline $2-3$ & 0.11 & 0.11 & 0.11 & 0.11 & restrained & $13-15-16$ & 100 & 100 & 96.98 & 93 & 25 \\
\hline $11-12$ & 0.11 & 0.11 & 0.11 & 0.11 & restrained & $13-15-18$ & 127 & 127 & 101.56 & 88 & 25 \\
\hline \multirow[t]{7}{*}{$8-9$} & 0.265 & 0.265 & 0.265 & 0.265 & restrained & $16-15-18$ & 100 & 100 & 166.35 & 172 & 25 \\
\hline & & & & & & $1-17-18$ & 127 & 127 & 145.19 & 134 & 25 \\
\hline & & & & & & $15-18-17$ & 127 & 127 & 127.03 & 129 & 25 \\
\hline & & & & & & 15-18-19 & 100 & 100 & 125.86 & 140 & 25 \\
\hline & & & & & & 17-18-19 & 100 & 100 & 98.17 & 98 & 25 \\
\hline & & & & & & $18-19-20$ & 180 & 180 & 180 & 180 & 25 \\
\hline & & & & & & $19-20-21$ & 180 & 180 & 180 & 180 & 25 \\
\hline
\end{tabular}


Table S4. Surfactin Micelle Size, Morphology, and Solvent Accessible Surface Area.

\begin{tabular}{llllll}
\hline & micelle diameter & & SAS of hydrophilic & \multicolumn{2}{l}{ SAS of hydrophobic } \\
system & $d(\AA)$ & ellipsoid $\alpha$ & part $A_{\text {phi }}\left(\mathrm{nm}^{2}\right)$ & part $A_{\text {pho }}\left(\mathrm{nm}^{2}\right)$ & $A_{\text {phi }} / A_{\text {pho }}$ \\
\hline $\mathrm{AM}$ & $45.9 \pm 0.2$ & $0.096 \pm 0.014$ & $175.7 \pm 2.4$ & $51.2 \pm 1.4$ & 3.43 \\
$\mathrm{CGM}_{\mathrm{I}}$ & $45.0 \pm 0.4$ & $0.115 \pm 0.051$ & $185.7 \pm 3.0$ & $52.4 \pm 1.5$ & 3.54 \\
$\mathrm{CGM}_{\text {II }}$ & $44.6 \pm 0.5$ & $0.129 \pm 0.042$ & $180.0 \pm 3.0$ & $51.3 \pm 1.5$ & 3.51 \\
$\mathrm{CGM}_{\mathrm{III}}$ & $44.9 \pm 0.4$ & $0.111 \pm 0.046$ & $185.8 \pm 3.0$ & $51.8 \pm 1.5$ & 3.59 \\
$\mathrm{CGM}_{\mathrm{IV}}$ & $44.4 \pm 0.4$ & $0.114 \pm 0.051$ & $179.3 \pm 2.9$ & $51.6 \pm 1.5$ & 3.47 \\
\hline
\end{tabular}


Table S5. Bond Interaction Parameters for CG Simulations of Pre-Assembled Micelle in Aqueous Solution.

\begin{tabular}{|c|c|c|c|c|c|c|c|c|c|c|c|}
\hline \multirow[b]{2}{*}{$i-j$} & \multirow[b]{2}{*}{$\mathrm{CGM}_{\mathrm{I}}$} & \multicolumn{2}{|c|}{$d_{\mathrm{ij}}(\mathrm{nm})$} & \multirow{2}{*}{\multicolumn{2}{|c|}{$K_{\mathrm{d}}(\mathrm{kJ} / \mathrm{mol})$}} & \multirow[b]{2}{*}{$i-j-k$} & \multicolumn{4}{|c|}{$\theta_{i j k}($ degree $)$} & \multirow[t]{2}{*}{$K_{\theta}(\mathrm{kJ} / \mathrm{mol})$} \\
\hline & & $\mathrm{CGM}_{\mathrm{II}}$ & $\mathrm{CGM}_{\mathrm{III}}$ & & & & $\mathrm{CGM}_{\mathrm{I}}$ & $\mathrm{CGM}_{\mathrm{II}}$ & $\mathrm{CGM}_{\mathrm{III}}$ & $\mathrm{CGM}_{\mathrm{IV}}$ & \\
\hline $1-2$ & 0.4 & 0.285 & 0.4 & 0.285 & 5000 & $2-1-4$ & 100 & 100 & 126.91 & 126.91 & 25 \\
\hline $1-4$ & 0.35 & 0.338 & 0.35 & 0.338 & 200 & $2-1-17$ & 100 & 100 & 97.97 & 97.97 & 25 \\
\hline $1-17$ & 0.321 & 0.321 & 0.321 & 0.321 & 1250 & $4-1-17$ & 127 & 127 & 114.25 & 114.25 & 25 \\
\hline $4-5$ & 0.33 & 0.33 & 0.33 & 0.33 & 7500 & $1-4-5$ & 100 & 100 & 92.18 & 92.18 & 25 \\
\hline $4-6$ & 0.35 & 0.31 & 0.35 & 0.31 & 200 & $1-4-6$ & 127 & 127 & 86.24 & 86.24 & 25 \\
\hline $6-7$ & 0.33 & 0.335 & 0.33 & 0.335 & 7500 & $5-4-6$ & 100 & 100 & 149.8 & 149.8 & 25 \\
\hline $6-8$ & 0.35 & 0.366 & 0.35 & 0.366 & 200 & $4-6-7$ & 100 & 100 & 99.79 & 99.79 & 25 \\
\hline $8-10$ & 0.35 & 0.33 & 0.35 & 0.33 & 200 & $4-6-8$ & 127 & 127 & 126.72 & 126.72 & 25 \\
\hline $10-11$ & 0.32 & 0.245 & 0.32 & 0.245 & 7500 & $7-6-8$ & 100 & 100 & 114.84 & 114.84 & 25 \\
\hline $10-13$ & 0.35 & 0.321 & 0.35 & 0.321 & 200 & $6-8-9$ & 100 & 100 & 98.46 & 98.46 & 25 \\
\hline $13-14$ & 0.33 & 0.333 & 0.33 & 0.333 & 7500 & $6-8-10$ & 127 & 127 & 93.83 & 93.83 & 25 \\
\hline $13-15$ & 0.35 & 0.358 & 0.35 & 0.358 & 200 & $9-8-10$ & 100 & 100 & 159.35 & 159.35 & 25 \\
\hline $15-16$ & 0.33 & 0.334 & 0.33 & 0.334 & 7500 & $8-10-11$ & 100 & 100 & 93.05 & 93.05 & 25 \\
\hline $15-18$ & 0.246 & 0.246 & 0.246 & 0.246 & 1250 & $8-10-13$ & 127 & 127 & 111.22 & 111.22 & 25 \\
\hline $17-18$ & 0.271 & 0.271 & 0.271 & 0.271 & 1250 & $11-10-13$ & 100 & 100 & 120.31 & 120.31 & 25 \\
\hline $18-19$ & 0.33 & 0.337 & 0.33 & 0.337 & 7500 & $10-13-14$ & 100 & 100 & 113.39 & 113.39 & 25 \\
\hline $19-20$ & 0.47 & 0.47 & 0.47 & 0.47 & 1250 & $10-13-15$ & 127 & 127 & 104.84 & 104.84 & 25 \\
\hline $20-21$ & 0.47 & 0.448 & 0.47 & 0.448 & 1250 & $14-13-15$ & 100 & 100 & 119.63 & 119.63 & 25 \\
\hline $2-3$ & 0.11 & 0.11 & 0.11 & 0.11 & restrained & $13-15-16$ & 100 & 100 & 91.11 & 91.11 & 25 \\
\hline $11-12$ & 0.11 & 0.11 & 0.11 & 0.11 & restrained & $13-15-18$ & 127 & 127 & 94.28 & 94.28 & 25 \\
\hline \multirow[t]{7}{*}{$8-9$} & 0.265 & 0.265 & 0.265 & 0.265 & restrained & $16-15-18$ & 100 & 100 & 166.35 & 166.35 & 25 \\
\hline & & & & & & $1-17-18$ & 127 & 127 & 152.84 & 152.84 & 25 \\
\hline & & & & & & $15-18-17$ & 127 & 127 & 134.18 & 134.18 & 25 \\
\hline & & & & & & $15-18-19$ & 100 & 100 & 122.6 & 122.6 & 25 \\
\hline & & & & & & $17-18-19$ & 100 & 100 & 97.25 & 97.25 & 25 \\
\hline & & & & & & $18-19-20$ & 180 & 180 & 180 & 180 & 25 \\
\hline & & & & & & $19-20-21$ & 180 & 180 & 180 & 180 & 25 \\
\hline
\end{tabular}



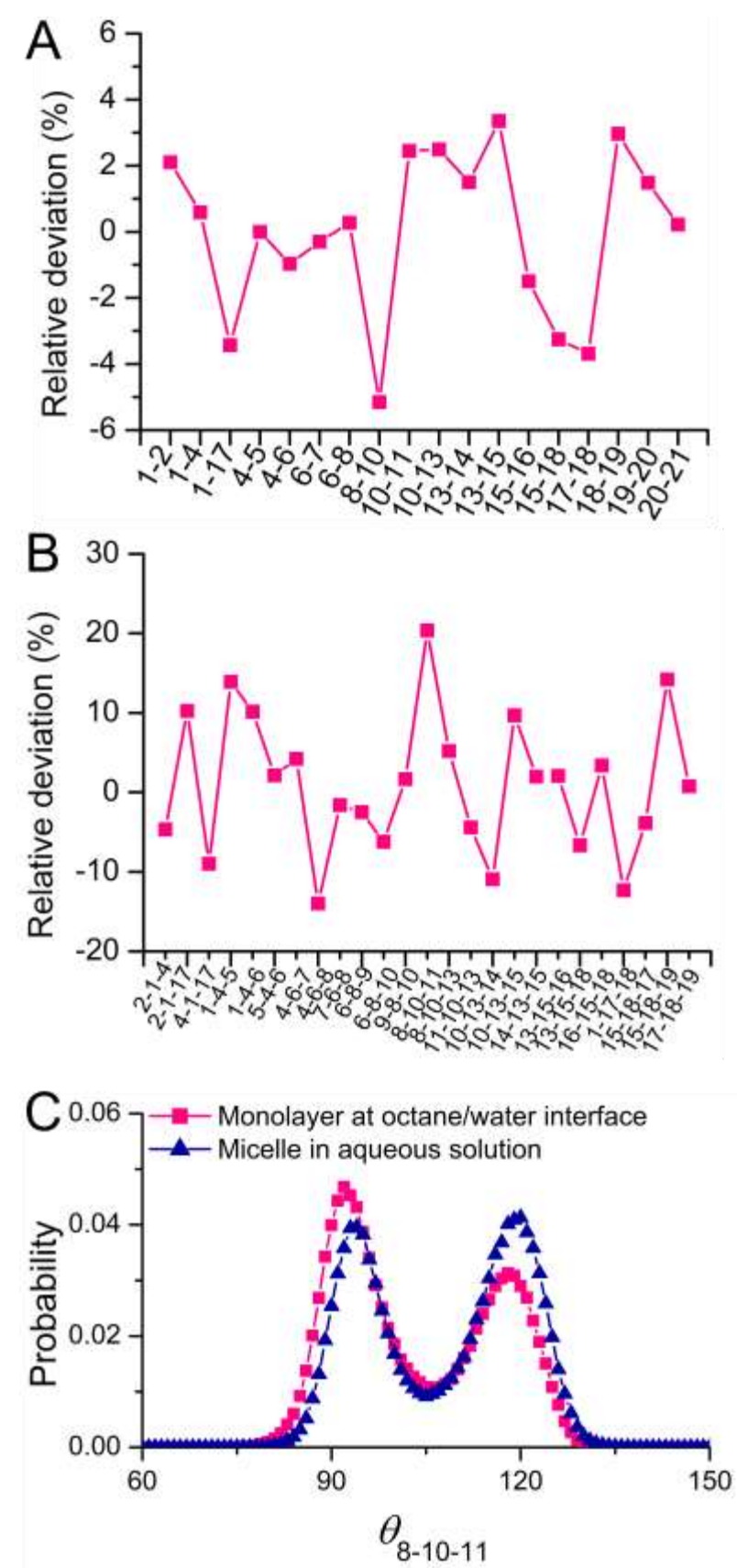

Figure S1. (A) Comparisons of bond lengths of surfactin within monolayer with those extracted from surfactin micelle in aqueous solution. (B) Comparisons of bond angles of surfactin within monolayer with those extracted from surfactin micelle in aqueous solution. (C) Multimodal distributions of one bond angle. 